\title{
Uplink Fractional Power Control for Cell-Free Wireless Networks
}

\author{
Rasoul Nikbakht, Angel Lozano \\ Universitat Pompeu Fabra (UPF) \\ 08018 Barcelona, Spain. \\ Email: \{rasoul.nikbakht, angel.lozano\}@upf.edu
}

\begin{abstract}
This paper proposes a power control policy for the uplink of cell-free wireless networks. Such policy, which generalizes the fractional power control used extensively in cellular networks, relies only on large-scale quantities, is fully distributed, and features a single control parameter. By adjusting this parameter, the SIR distribution experienced by the users can be compressed or expanded, effecting a tradeoff between average performance and fairness.
\end{abstract}

\section{INTRODUCTION}

Cell-free networks can be regarded as a deconstruction of cellular massive MIMO whereby the many antennas that would be collocated at cell sites are scattered over the network, and the associations between users and cells are released. What results is a dense infrastructure of access points (APs), each featuring one or a few antennas, with every user potentially communicating with every AP [1]-[5]. Alternatively, this can be seen as a simplified form of network MIMO [6]-[8] or of centralized radio access [9], with substantially more antennas than users per time-frequency signaling resource so as to render matched-filter beamforming effective. At the expense of extensive backhaul, cell-free networks offer multiple advantages over their cellular counterparts, including large-scale diversity and reduced distances to the end users.

Concentrating on the uplink, one of the challenges that arises is that of power control. Given the major differences in pathloss and shadowing among links, large-scale power control appears essential to keep those users near an AP from overwhelming the rest and to avoid huge performance disparities. The effectiveness of power control is illustrated in [1], where it is shown that a max-min solution for the user transmit powers does yield a highly equalized performance across the network. While encouraging, this solution is not an implementably convenient option because it must be found in an iterative and centralized fashion, meaning that it is not distributed and it does not scale. Also, it does not offer the possibility of regulating the degree of performance equalization, something desirable to trade average performance for fairness.

The desiderata for a power control policy that can be effectively implemented are:

- To retain the virtue, exhibited by the max-min solution in [1], of depending only on large-scale quantities.

- To be fully distributed, meaning that every user controls its own power based only on its own channel gains to the network.
- To allow regulating the extent to which the performance is equalized across users.

The challenge of devising such a policy was tackled in due time for cellular networks, and a satisfying answer was found in the form of fractional power control; variations of this approach have been adopted by standards, including LTE and NR. Our goal is to extend the fractional power control formulation to cell-free networks, proposing a policy that satisfies the foregoing desiderata.

\section{NETWORK AND CHANNEL MODELS}

The networks under consideration feature $N$ single-antenna APs and $K$ single-antenna users, with all antennas omnidirectional in azimuth. Every user can communicate with every AP on each time-frequency resource. Some of the uplink resources are reserved for pilot transmissions, based on which the channels are estimated by the APs.

\section{A. Large-scale Modeling}

Provided the AP locations are agnostic to the radio propagation, shadowing has been shown to make those locations appear Poisson-distributed from the vantage of any user [10]. Although asymptotic in the shadowing strength, this phenomenon is well manifested for shadowing intensities of interest [10]-[12]. Capitalizing on this result, we draw the AP positions uniformly, avoiding the need for explicit modeling of the shadowing as it is then already implicitly captured by the network geometry. Likewise, the user positions are drawn uniformly. Altogether, the locations of APs and users conform to (independent) binomial point processes; as the network grows large, these converge to Poisson point processes.

The operating bandwidth is in the microwave range. Signals are subject to pathloss with exponent $\eta$, giving a localaverage channel gain $G_{n, k}$ between the $k$ th user and the $n$th AP. The corresponding local-average SNR equals $\mathrm{SNR}_{n, k}=$ $G_{n, k} P / \sigma^{2}$ with $P$ the maximum transmit power measured at $1 \mathrm{~m}$ from the user, so that no scaling constants are needed, and with $\sigma^{2}$ the noise power within the signal bandwidth. The local-average SNRs, and the large-scale parameters in general, are stable and known for each network snapshot.

\section{B. Small-scale Modeling}

Besides $G_{n, k}$, the channel between the $k$ th user and the $n$th AP features a small-scale fading coefficient $h_{n, k} \sim \mathcal{N}_{\mathbb{C}}(0,1)$, independent across users and APs. 


\section{Cell-Free Uplink Formulation}

\section{A. Reverse-link Channel Estimation}

Let $\mathcal{P}_{k}$ be the set of users (including user $k$ ) that share the pilot of user $k$. Orthogonal pilots are assigned to different sets. The simultaneous transmission from the users in set $\mathcal{P}_{k}$ of a pilot of power $P$ is observed at the $n$th AP as

$$
y_{n}=\sum_{\mathrm{k} \in \mathcal{P}_{k}} \sqrt{G_{n, \mathrm{k}}} h_{n, \mathrm{k}} \sqrt{P}+v_{n}
$$

where $v_{n} \sim \mathcal{N}_{\mathbb{C}}\left(0, \sigma^{2}\right)$. From $y_{n}$, the $n$th AP produces the LMMSE channel estimate $\hat{h}_{n, k}$ satisfying $h_{n, k}=\hat{h}_{n, k}+\tilde{h}_{n, k}$ where $\tilde{h}_{n, k} \sim \mathcal{N}_{\mathbb{C}}\left(0, \mathrm{MMSE}_{n, k}\right)$ is uncorrelated error and

$$
\operatorname{MMSE}_{n, k}=\frac{1+\sum_{\mathrm{k} \in \mathcal{P}_{k}, \mathrm{k} \neq k} \mathrm{SNR}_{n, \mathrm{k}}}{1+\sum_{\mathrm{k} \in \mathcal{P}_{k}} \operatorname{SNR}_{n, \mathrm{k}}} .
$$

As the problem we address is not directly related to pilot contamination, and since there are ways of keeping it at bay in cell-free networks [1], [13]-[15], to avoid distractions and the need to posit specific pilot assignment schemes, we disregard the contamination. That amounts to $\mathcal{P}_{k}$ containing only user $k$, meaning that

$$
\begin{aligned}
\mathbb{E}\left[\left|\hat{h}_{n, k}\right|^{2}\right] & =\frac{\mathrm{SNR}_{n, k}}{1+\mathrm{SNR}_{n, k}} \\
\mathbb{E}\left[\left|\tilde{h}_{n, k}\right|^{2}\right] & =\frac{1}{1+\mathrm{SNR}_{n, k}} .
\end{aligned}
$$

\section{B. Data Transmission}

Upon payload data transmission, the $n$th AP observes

$$
y_{n}=\sum_{k=0}^{K-1} \sqrt{G_{n, k}} h_{n, k} \sqrt{p_{k} P} s_{k}+v_{n},
$$

where $s_{k}$ is the unit-variance symbol transmitted by user $k$ while $p_{k} \in[0,1]$ is its power control coefficient.

With matched filtering, the signal of user $k$ is recovered as

$$
\sum_{n=0}^{N-1} w_{n, k}^{*} y_{n}
$$

where $w_{n, k}=\sqrt{G_{n, k}} \hat{h}_{n, k}$. This gives

$$
\begin{aligned}
\sum_{n=0}^{N-1} w_{n, k}^{*} y_{n}= & \underbrace{\sum_{n=0}^{N-1} G_{n, k} \sqrt{p_{k} P} \hat{h}_{n, k}^{*} \hat{h}_{n, k} s_{k}}_{\text {Desired Signal: } S_{k}} \\
& +\underbrace{\sum_{n=0}^{N-1} \sqrt{G_{n, k} P} \hat{h}_{n, k}^{*} \sum_{\mathrm{k} \neq k} \sqrt{G_{n, \mathrm{k}}} \sqrt{p_{\mathrm{k}}} \hat{h}_{n, \mathrm{k}} s_{\mathrm{k}}}_{\text {Multiuser Interference: } I_{k}} \\
& +\underbrace{\sum_{n=0}^{N-1} \sqrt{G_{n, k} P} \hat{h}_{n, k}^{*} \sum_{\mathrm{k}=0}^{K-1} \sqrt{G_{n, \mathrm{k}}} \sqrt{p_{\mathrm{k}}} \tilde{h}_{n, \mathrm{k}} s_{\mathrm{k}}}_{\text {Channel Estimation Errors: } E_{k}} \\
& +\underbrace{\sum_{n=0}^{N-1} \sqrt{G_{n, k}} \hat{h}_{n, k}^{*} v_{n}}_{\text {Filtered Noise: } V_{k}},
\end{aligned}
$$

from which the signal-to-interference-plus-noise ratio (SINR) of user $k$, conditioned on the known $\left\{\hat{h}_{n, k}\right\}_{n=0}^{N-1}$, equals

$$
\begin{aligned}
\operatorname{sinr}_{k} & =\frac{\mathbb{E}\left[\left|S_{k}\right|^{2} \mid\left\{\hat{h}_{n, k}\right\}_{n=0}^{N-1}\right]}{\mathbb{E}\left[\left|I_{k}\right|^{2}+\left|E_{k}\right|^{2}+\left|V_{k}\right|^{2} \mid\left\{\hat{h}_{n, k}\right\}_{n=0}^{N-1}\right]} \\
& =\frac{P\left(\sum_{n=0}^{N-1} G_{n, k} \sqrt{p_{k}}\left|\hat{h}_{n, k}\right|^{2}\right)^{2}}{\operatorname{den}_{k}}
\end{aligned}
$$

with

$$
\begin{aligned}
\operatorname{den}_{k}= & P \sum_{\mathrm{k} \neq k} p_{\mathrm{k}}\left|\sum_{n=0}^{N-1} \sqrt{G_{n, k} G_{n, \mathrm{k}}} \hat{h}_{n, k}^{*} \hat{h}_{n, \mathrm{k}}\right|^{2} \\
& +P \sum_{\mathrm{k}=0}^{K-1} p_{\mathrm{k}} \sum_{n=0}^{N-1} G_{n, k} G_{n, \mathrm{k}}\left|\hat{h}_{n, k}\right|^{2} \mathrm{MMSE}_{n, \mathrm{k}} \\
& +\sum_{n=0}^{N-1} G_{n, k}\left|\hat{h}_{n, k}\right|^{2} \sigma^{2} .
\end{aligned}
$$

After some simplifications, and substituting for $\mathrm{MMSE}_{n, k}$,

$$
\operatorname{sinr}_{k}=\frac{p_{k}\left(\sum_{n=0}^{N-1} \mathrm{SNR}_{n, k}\left|\hat{h}_{n, k}\right|^{2}\right)^{2}}{\operatorname{den}_{k}}
$$

with

$$
\begin{aligned}
\operatorname{den}_{k}= & \sum_{\mathrm{k} \neq k} p_{\mathrm{k}}\left|\sum_{n=0}^{N-1} \sqrt{\mathrm{SNR}_{n, k} \mathrm{SNR}_{n, \mathrm{k}}} \hat{h}_{n, k}^{*} \hat{h}_{n, \mathrm{k}}\right|^{2} \\
& +\sum_{\mathrm{k}=0}^{K-1} p_{\mathrm{k}} \sum_{n=0}^{N-1} \frac{\mathrm{SNR}_{n, k} \mathrm{SNR}_{n, \mathrm{k}}}{1+\mathrm{SNR}_{n, \mathrm{k}}}\left|\hat{h}_{n, k}\right|^{2} \\
& +\sum_{n=0}^{N-1} \mathrm{SNR}_{n, k}\left|\hat{h}_{n, k}\right|^{2} .
\end{aligned}
$$

In interference-limited conditions, (11)-(12) simplify into

$$
\operatorname{sir}_{k}=\frac{p_{k}\left(\sum_{n=0}^{N-1} G_{n, k}\left|h_{n, k}\right|^{2}\right)^{2}}{\sum_{\mathrm{k} \neq k} p_{\mathrm{k}}\left|\sum_{n=0}^{N-1} \sqrt{G_{n, k} G_{n, \mathrm{k}}} h_{n, k}^{*} h_{n, \mathrm{k}}\right|^{2}} .
$$

\section{Fractional Power CONTROL}

\section{A. Description for Cellular Networks}

The origins of fractional power control, which, as mentioned, is the mainstream approach in cellular networks, can be traced back to [16]. In that initial derivation, the goal was to minimize the variance of the large-scale SIR distribution (in $\mathrm{dB}$ ) experienced by two interfering cellular users, and the found solution was $p_{k} \propto 1 / \sqrt{G_{k}}$ where $G_{k}$ is the large-scale gain to the serving cell and the proportionality is such that $p_{k} \in[0,1]$. This was subsequently generalized to [17], [18]

$$
p_{k} \propto \frac{1}{G_{k}^{\vartheta}}
$$

where $\vartheta \in[0,1]$ regulates the extent to which the range of $\mathrm{dB}$-scale received powers is compressed. The values typically featured in LTE are in the range $\vartheta \approx 0.5-0.7$, with lower values favoring the average SIR while higher values promote more fairness and better cell-edge performance. 


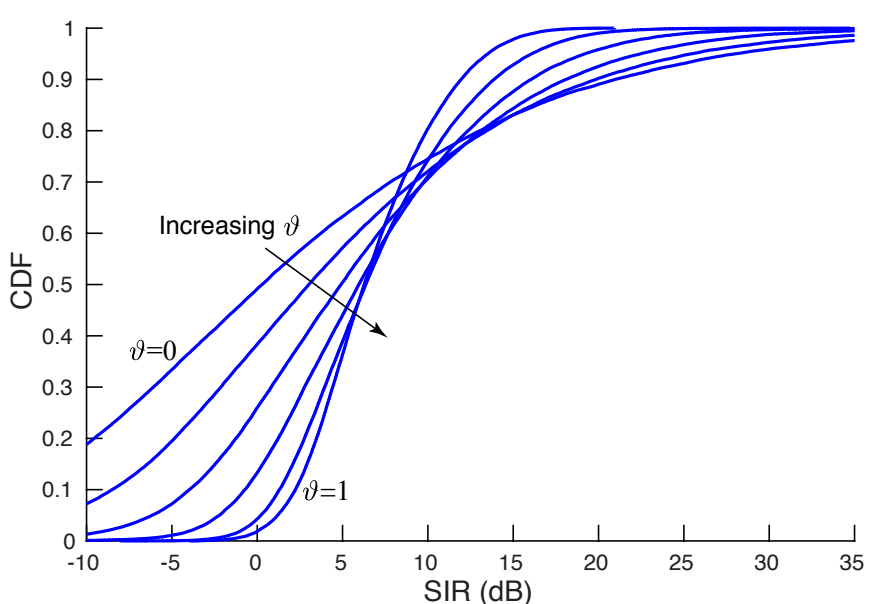

Fig. 1: CDF of SIR (in $\mathrm{dB}$ ) for $\eta=3.8$ and $N / K=2.5$, parameterized by $\vartheta=\{0,0.2,0.4,0.6,0.8,1\}$.

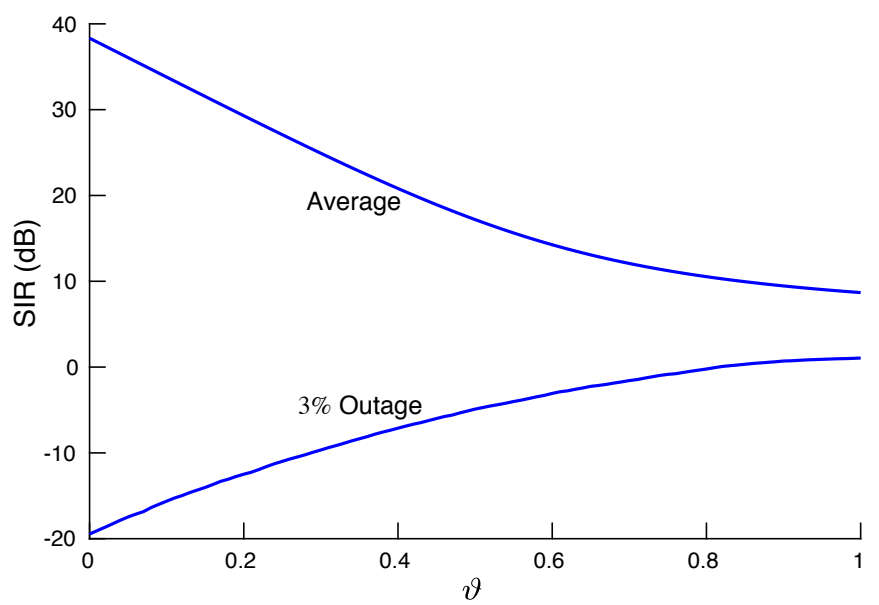

Fig. 2: Average and 3\%-outage SIRs (in $\mathrm{dB}$ ) as a function of $\vartheta$, for $\eta=3.8$ and $N / K=2.5$.

\section{B. Generalization to Cell-Free Networks}

Our proposed generalization of (14) to cell-free networks, derived in Appendix A, is

$$
p_{k} \propto \frac{1}{\left(\sum_{n=0}^{N-1} G_{n, k}\right)^{\vartheta}},
$$

which does reduce to (14) if users connect to only $N=1 \mathrm{AP}$, or if the gain to one of the APs markedly dominates the rest. More broadly, the power control now depends on all the largescale channel gains that involve a given user, reflecting the effective connection between such user and the entire network when matched-filter beamforming is applied.

In the next section, we proceed to evaluate the performance of the power control policy in (15).

\section{Some Results}

To exemplify the performance of the proposed policy, we consider a wrapped-around universe with $N=200$ APs and a pathloss exponent of $\eta=3.8$. Each CDF corresponds to

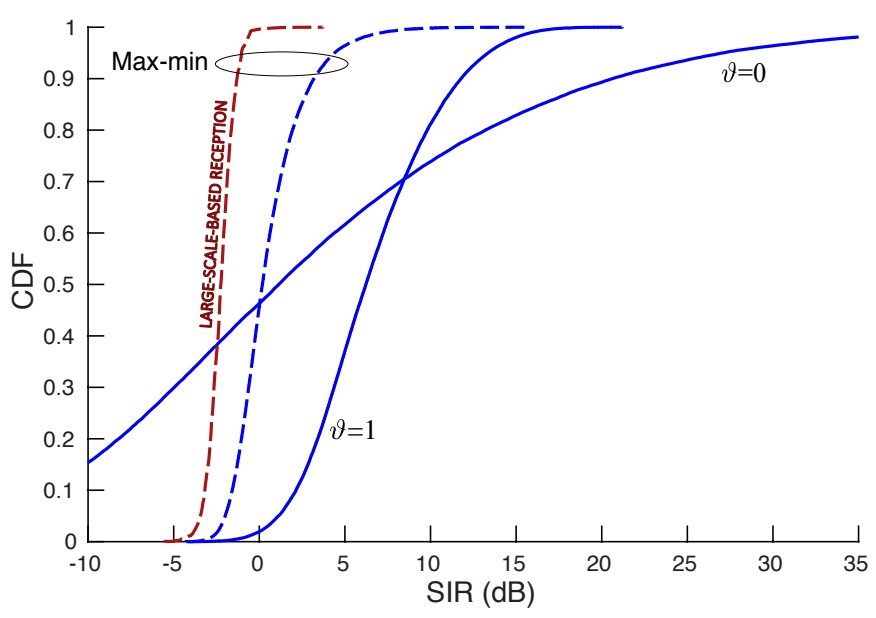

Fig. 3: Proposed fractional power control policy (with $\vartheta=0$ and $\vartheta=1$ ) against the max-min solution, for $\eta=3.8$ and $N / K=2.5$. The max-min solution is shown for both channel-estimation-based and large-scale-based reception.

500 network snapshots, ensuring that with $95 \%$ confidence the CDF does not deviate from its true value by more than $0.3 \%$. Thanks to the wrap-around, all APs contribute equally to the statistics. The presented SIRs are local-averages, meaning that they have been expected over the small-scale fading.

Shown in Fig. 1 is the CDF of the SIR (in $\mathrm{dB}$ ) for $N / K=2.5$, parameterized by the fractional power control parameter $\vartheta$. By sweeping this parameter from $\vartheta=0$ (fixed transmit powers) to the maximum value of $\vartheta=1$, we observe a progressive compression of the $\mathrm{CDF}$ and a reduction of its variance.

The tradeoff that $\vartheta$ enables between the average SIR and the lower tail (as a proxy for fairness) can be appreciated in Fig. 2, which depicts, again for $N / K=2.5$, the average SIR and the $3 \%$-outage SIR (i.e., the SIR achieved by $97 \%$ of users) as a function of $\vartheta$. As we move from $\vartheta=0$ to $\vartheta=1$, the 3\%-outage SIR increases steadily at the expense of the average. Having the $3 \%$-outage SIR at a reasonable $-5 \mathrm{~dB}$, for instance, would entail $\vartheta \approx 0.5$.

Then, Fig. 3 contrasts the proposed policy with the max-min solution. Such max-min solution involves the SIR achieved with large-scale-based reception, namely [1]

$$
\mathrm{SIR}_{k}=\frac{p_{k}\left(\sum_{n=0}^{N-1} G_{n, k}\right)^{2}}{\sum_{\mathrm{k}=0}^{K-1} p_{\mathrm{k}} \sum_{n=0}^{N-1} G_{n, k} G_{n, \mathrm{k}}},
$$

which relies on channel hardening rather than on channel estimates. The $\left\{p_{k}\right\}_{k=0}^{K-1}$ that maximize $\min \left(\left\{\mathrm{SIR}_{k}\right\}_{k=0}^{K-1}\right)$ are obtained iteratively as per [1, Sec. IV], plugged into (13), and expected over the small-scale fading for each network snapshot. (For the sake of completeness, Fig. 3 also depicts the distribution of $\mathrm{SIR}_{k}$ itself.) What we observe in the figure is that the SIR distribution with fractional power control uniformly exceeds the max-min solution, even if the former does not become as steep as the latter. The max-min solution appears to be dragged down by worst-case users, paying a 


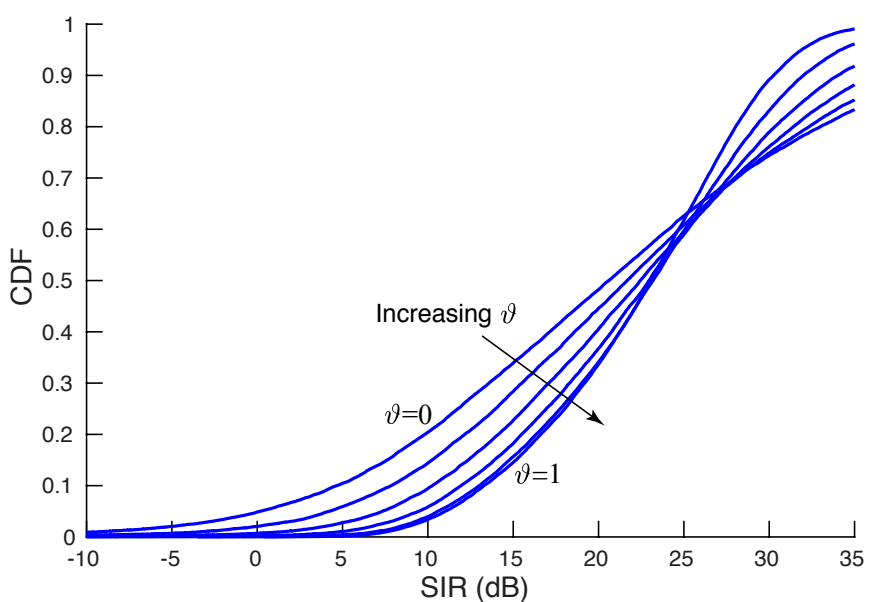

Fig. 4: CDF of SIR (in $\mathrm{dB}$ ) for $\eta=3.8$ and $N / K=10$, parameterized by $\vartheta=\{0,0.2,0.4,0.6,0.8,1\}$.

substantial price for its uncompromising equalization of all SIRs, while fractional power control benefits from being more ductile.

The set of results presented in Figs. $1-3$ for $N / K=2.5$ is reproduced, for a more conservative ratio of $N / K=10$, in Figs. 4-6. The behaviors are qualitatively similar, only with a reduced sensitivity to the power control parameter $\vartheta$; in this case, a $3 \%$-outage SIR of $-5 \mathrm{~dB}$ would entail $\vartheta \approx 0.4$. The proposed policy continues to compare very favorably with the max-min solution.

\section{SUMMARY}

The proposed fractional power control policy, embodied by (15), satisfies the desiderata of depending only on large-scale quantities (an additional small-scale power control loop could thus be overlaid), of being fully distributed (each user controls its power based only on its own channel gains), and of being manageable through a single parameter. Its performance is highly satisfactory.

The potential follow-ups to this work include the generalization to settings where noise is not negligible, and a power allocation policy for the cell-free downlink.

\section{ACKNOWLEDGMENT}

Work supported by the Spanish Ministry of Economy and Competitiveness under the Maria de Maeztu Units of Excellence Programme (MDM-2015-0502) and by the European Research Council (grant agreement 694974).

\section{APPENDIX A}

DERIVATION OF (15)

Following on the footsteps of [16], we seek to minimize the variance of the large-scale SIR distribution (in $\mathrm{dB}$ ), hence the starting point needs to be an expression for such SIR. In a cellular network, this equals the local-average desired signal

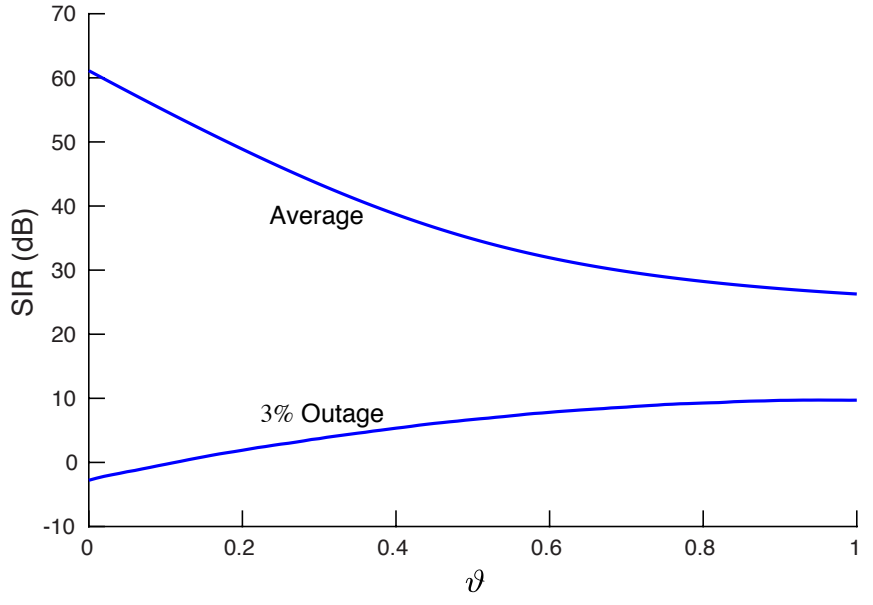

Fig. 5: Average and 3\%-outage SIRs (in $\mathrm{dB}$ ) as a function of $\vartheta$, for $\eta=3.8$ and $N / K=10$.

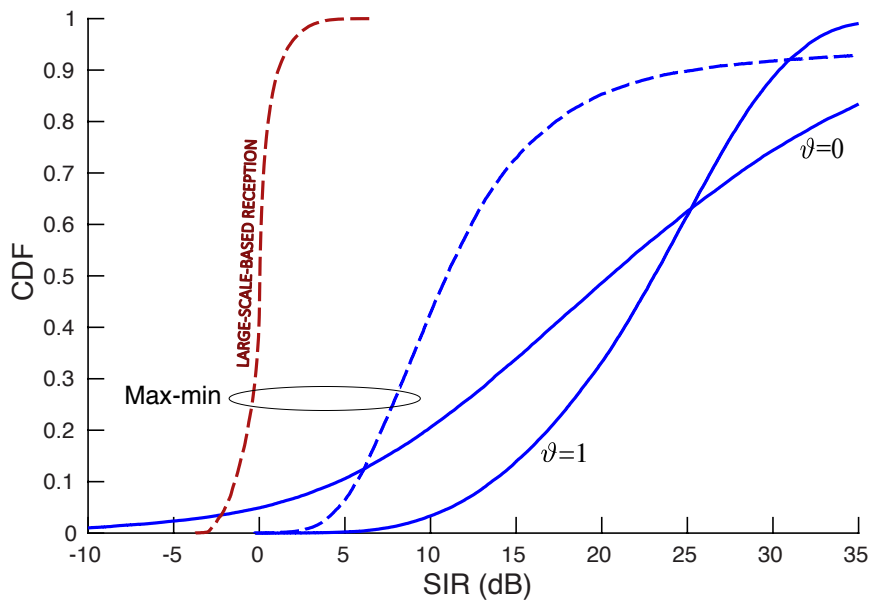

Fig. 6: Proposed fractional power control policy (with $\vartheta=0$ and $\vartheta=1$ ) against the max-min solution, for $\eta=3.8$ and $N / K=10$. The max-min solution is shown for both channel-estimation-based and large-scale-based reception.

power divided by the local-average other-cell interference power, namely [19]

$$
\mathrm{SIR}_{k}=\frac{p_{k} G_{k}}{\sum_{\mathrm{k} \neq k} p_{\mathrm{k}} G_{\mathrm{k}}}
$$

which can be recovered from (13) by letting $N=1$ and replacing $\left|h_{k}\right|^{2}$ and $\left|h_{\mathrm{k}}\right|^{2}$ by their unit expected value. A counterpart to (17) for cell-free networks can be obtained by similarly replacing $\left|h_{n, k}\right|^{2}$ and $\left|h_{n, \mathrm{k}}\right|^{2}$ by unity, and by zeroing out cross-terms containing $h_{n, k}^{*} h_{n, \mathrm{k}}$ in the expansion of the denominator. This gives

$$
\mathrm{SIR}_{k}=\frac{p_{k}\left(\sum_{n=0}^{N-1} G_{n, k}\right)^{2}}{\sum_{\mathrm{k} \neq k} p_{\mathrm{k}} \sum_{n=0}^{N-1} G_{n, k} G_{n, \mathrm{k}}} .
$$

As a side note we point out that, interestingly, (18) is almost identical to the SIR that would be achieved with channelhardening-based reception [1, Eq. 27], i.e., if the network 
regarded $\sum_{n=0}^{N-1} \mathbb{E}\left[w_{n, k}^{*} y_{n}\right]$ rather than $\sum_{n=0}^{N-1} w_{n, k}^{*} y_{n}$ as the desired signal, with everything else regarded as interference. As only difference, channel-hardening-based reception would generate the self-interference

$$
\sum_{n=0}^{N-1}\left(w_{n, k}^{*} y_{n}-\mathbb{E}\left[w_{n, k}^{*} y_{n}\right]\right),
$$

which would extend the SIR denominator summation in (18) to include $\mathrm{k}=k$ (cf. Eq. 16).

Taking (18) as it stands, we can specialize it to $K=2$ and focus, without loss of generality, on user 0 . That yields

$$
\mathrm{SIR}_{0}=\frac{p_{0}\left(\sum_{n=0}^{N-1} G_{n, 0}\right)^{2}}{p_{1} \sum_{n=0}^{N-1} G_{n, 0} G_{n, 1}},
$$

from which, introducing the notation $\left.z\right|_{\mathrm{dB}}=10 \log _{10} z$, we can further write

$$
\left.\mathrm{SIR}_{0}\right|_{\mathrm{dB}}=\left.p_{0}\right|_{\mathrm{dB}}+\left.2 G_{\mathrm{S}}\right|_{\mathrm{dB}}-\left.p_{1}\right|_{\mathrm{dB}}-\left.G_{\mathrm{I}}\right|_{\mathrm{dB}},
$$

where $G_{\mathrm{S}}=\sum_{n=0}^{N-1} G_{n, 0}$ and $G_{\mathrm{I}}=\sum_{n=0}^{N-1} G_{n, 0} G_{n, 1}$.

We are interested in distributed power control policies where $p_{0}$ depends on $\left\{G_{n, 0}\right\}_{n=0}^{N-1}$, but not on $\left\{G_{n, 1}\right\}_{n=0}^{N-1}$, hence the following can be reasonably assumed to hold:

- $p_{0}$ and $p_{1}$ are independent, as they are controlled on the basis of distinct channel gains, with independent shadowings.

- $p_{0}$ is not independent of $G_{\mathrm{S}}$, nor of $G_{\mathrm{I}}$.

- $p_{1}$ is not independent of $G_{\mathrm{I}}$, but it is independent of $G_{\mathrm{S}}$. With that taken into account, the variance of (21) can be developed into

$$
\begin{aligned}
\operatorname{var}\left[\left.\operatorname{SIR}_{0}\right|_{\mathrm{dB}}\right]= & \operatorname{var}\left[\left.p_{0}\right|_{\mathrm{dB}}+\left.2 G_{\mathrm{S}}\right|_{\mathrm{dB}}\right]+\operatorname{var}\left[\left.p_{1}\right|_{\mathrm{dB}}+\left.G_{\mathrm{I}}\right|_{\mathrm{dB}}\right] \\
& -2 \operatorname{cov}\left[\left.p_{0}\right|_{\mathrm{dB}}+\left.2 G_{\mathrm{S}}\right|_{\mathrm{dB}},\left.p_{1}\right|_{\mathrm{dB}}+\left.G_{\mathrm{I}}\right|_{\mathrm{dB}}\right] \\
= & \operatorname{var}\left[\left.p_{0}\right|_{\mathrm{dB}}\right]+4 \operatorname{var}\left[\left.G_{\mathrm{S}}\right|_{\mathrm{dB}}\right]+4 \operatorname{cov}\left[\left.p_{0}\right|_{\mathrm{dB}},\left.G_{\mathrm{S}}\right|_{\mathrm{dB}}\right] \\
& +\operatorname{var}\left[\left.p_{1}\right|_{\mathrm{dB}}\right]+\operatorname{var}\left[\left.G_{\mathrm{I}}\right|_{\mathrm{dB}}\right]+2 \operatorname{cov}\left[\left.p_{1}\right|_{\mathrm{dB}},\left.G_{\mathrm{I}}\right|_{\mathrm{dB}}\right] \\
& -2 \operatorname{cov}\left[\left.p_{0}\right|_{\mathrm{dB}},\left.G_{\mathrm{I}}\right|_{\mathrm{dB}}\right]-4 \operatorname{cov}\left[\left.G_{\mathrm{S}}\right|_{\mathrm{dB}},\left.G_{\mathrm{I}}\right|_{\mathrm{dB}}\right],
\end{aligned}
$$

which, by virtue of the fact that $\operatorname{var}\left[\left.p_{0}\right|_{\mathrm{dB}}\right]=\operatorname{var}\left[\left.p_{1}\right|_{\mathrm{dB}}\right]$ and $\operatorname{cov}\left[\left.p_{0}\right|_{\mathrm{dB}}, G_{\mathrm{I}}\right]=\operatorname{cov}\left[\left.p_{1}\right|_{\mathrm{dB}}, G_{\mathrm{I}}\right]$, simplifies into

$$
\begin{aligned}
& \operatorname{var}\left[\left.\operatorname{SIR}_{0}\right|_{\mathrm{dB}}\right]=2 \operatorname{var}\left[\left.p_{0}\right|_{\mathrm{dB}}\right]+4 \operatorname{cov}\left[\left.p_{0}\right|_{\mathrm{dB}},\left.G_{\mathrm{S}}\right|_{\mathrm{dB}}\right] \\
& +4 \operatorname{var}\left[\left.G_{\mathrm{S}}\right|_{\mathrm{dB}}\right]+\operatorname{var}\left[\left.G_{\mathrm{I}}\right|_{\mathrm{dB}}\right]-4 \operatorname{cov}\left[\left.G_{\mathrm{S}}\right|_{\mathrm{dB}},\left.G_{\mathrm{I}}\right|_{\mathrm{dB}}\right] .
\end{aligned}
$$

Regrouping some terms, the above can be rewritten as

$$
\begin{aligned}
\operatorname{var}\left[\left.\mathrm{SIR}_{0}\right|_{\mathrm{dB}}\right]= & 2 \operatorname{var}\left[\left.p_{0}\right|_{\mathrm{dB}}+\left.G_{\mathrm{S}}\right|_{\mathrm{dB}}\right]+2 \operatorname{var}\left[\left.G_{\mathrm{S}}\right|_{\mathrm{dB}}\right] \\
& +\operatorname{var}\left[\left.G_{\mathrm{I}}\right|_{\mathrm{dB}}\right]-4 \operatorname{cov}\left[\left.G_{\mathrm{S}}\right|_{\mathrm{dB}},\left.G_{\mathrm{I}}\right|_{\mathrm{dB}}\right],
\end{aligned}
$$

where the last three terms do not depend on $\left.p_{0}\right|_{\mathrm{dB}}$, hence they are immaterial to the minimization with respect to it. The quantity to minimize is $\operatorname{var}\left[\left.p_{0}\right|_{\mathrm{dB}}+\left.G_{\mathrm{S}}\right|_{\mathrm{dB}}\right]$, and the power control policy that minimizes it is $\left.p_{0}\right|_{\mathrm{dB}}=-\left.G_{\mathrm{S}}\right|_{\mathrm{dB}}$, i.e.,

$$
p_{0}=\frac{1}{\sum_{n=0}^{N-1} G_{n, 0}} .
$$

It is interesting to note that, whereas in the two-user cellular case the SIR variance is minimized by the square-root policy $p_{0}=1 / \sqrt{G_{0}}$ [16], in the two-user cell-free case it is minimized by the full inversion policy in (24). This is because, in the former case, every user is detected by a distinct AP (the one in the serving cell) while, in the latter case, both users are detected by the same set of APs (all the APs in the network).

Finally, and with a view to having $K>2$ users and to regulating the forcefulness of the power control, we generalize (24) into (15), by introducing $\vartheta \in[0,1]$.

\section{REFERENCES}

[1] H. Q. Ngo, A. Ashikhmin, H. Yang, E. G. Larsson, and T. L. Marzetta, "Cell-free massive MIMO versus small cells," IEEE Trans. on Wireless Communications, vol. 16, no. 3, pp. 1834-1850, Mar. 2017.

[2] G. Interdonato, H. Q. Ngo, E. G. Larsson, and P. Frenger, "How much do downlink pilots improve cell-free massive MIMO?" in IEEE Global Communications Conf. (GLOBECOM'16), 2016, pp. 1-7.

[3] E. Nayebi, A. Ashikhmin, T. L. Marzetta, H. Yang, and B. D. Rao, "Precoding and power optimization in cell-free massive MIMO systems," IEEE Trans. Wireless Commun., vol. 16, pp. 4445-4459, Jul. 2017.

[4] E. Nayebi, A. Ashikhmin, T. L. Marzetta, and B. D. Rao, "Performance of cell-free massive MIMO systems with MMSE and LSFD receivers," in Asilomar Conf. Signals, Systems and Comp., Nov. 2016, pp. 203-207.

[5] H. Q. Ngo, L.-N. Tran, T. Q. Duong, M. Matthaiou, and E. G. Larsson, "On the total energy efficiency of cell-free massive MIMO," IEEE Trans. Green Commun. Networking, vol. 2, no. 1, pp. 25-39, Mar. 2018.

[6] M. K. Karakayali, G. J. Foschini, and R. A. Valenzuela, "Network coordination for spectrally efficient communications in cellular systems," IEEE Wireless Commun. Mag., vol. 3, no. 14, pp. 56- 61, Aug. 2006.

[7] S. Venkatesan, A. Lozano, and R. Valenzuela, "Network MIMO: Overcoming intercell interference in indoor wireless systems," Asilomar Conf. Signals, Systems and Computers, pp. 83-87, 2007.

[8] S. Venkatesan, H. Huang, A. Lozano, and R. Valenzuela, "A WiMAXbased implementation of network MIMO for indoor wireless systems," EURASIP J. Adv. Signal Processing, vol. 2009, Sep. 2009.

[9] A. Checko et al., "Cloud RAN for mobile networks - a technology overview," IEEE Communications Surveys \& Tutorials, vol. 17, no. 1, pp. 405-426, 1st Quart. 2015.

[10] B. Błaszczyszyn, M. K. Karray, and H. P. Keeler, "Wireless networks appear Poissonian due to strong shadowing," IEEE Trans. Wireless Communications, vol. 14, no. 8, pp. 4379-4390, Aug. 2015.

[11] G. George, R. K. Mungara, A. Lozano, and M. Haenggi, "Ergodic spectral efficiency in MIMO cellular networks," IEEE Trans. Wireless Commun., vol. 16, no. 5, pp. 2835-2849, May 2017.

[12] G. George, A. Lozano, and M. Haenggi, "Distribution of the number of users per base station in cellular networks," IEEE Wireless Commun. Letters, Jan. 2019.

[13] O. Y. Bursalioglu, C. Wang, H. Papadopoulos, and G. Caire, "RRH based massive MIMO with 'on the fly' pilot contamination control," in IEEE Int'l Conf. on Communications (ICC'16), 2016, pp. 1-7.

[14] - "A novel alternative to cloud RAN for throughput densification: Coded pilots and fast user-packet scheduling at remote radio heads," in Asilomar Conference on Signals, Systems and Computers, Nov. 2016, pp. 3-10.

[15] M. Attarifar, A. Abbasfar, and A. Lozano, "Random vs structured pilot assignment in cell-free massive MIMO wireless networks," IEEE Int'l Conf. Commun. Workshops (ICCW'18), May 2018.

[16] J. F. Whitehead, "Signal-level-based dynamic power control for cochannel interference management," in IEEE 43rd Vehicular Technology Conference, May 1993, pp. 499-502.

[17] R. D. Yates, "A framework for uplink power control in cellular radio systems," IEEE J. Sel. Areas Commun., vol. 13, no. 7, pp. 1341-1347, Sep. 1995.

[18] A. Simonsson and A. Furuskar, "Uplink power control in LTEoverview and performance," IEEE Veh. Techn. Conf. (VTC'08 Fall), pp. 1-5, Sep. 2008.

[19] R. W. Heath and A. Lozano, Foundations of MIMO Communication. Cambridge University Press, 2019. 